\title{
Hall coefficient and magnetoresistance of 2D spin-polarized electron systems
}

\author{
E. H. Hwang and S. Das Sarma \\ Condensed Matter Theory Center, Department of Physics, University of Maryland, College Park, Maryland 20742-4111
}

(February 2, 2020)

\begin{abstract}
Recent measurements of the 2D Hall resistance show that the Hall coefficient is independent of the applied in-plane magnetic field, i.e., the spin-polarization of the system. We calculate the weak-field Hall coefficient and the magnetoresistance of a spin polarized 2D system using the semi-classical transport approach based on the screening theory. We solve the coupled kinetic equations of the two carrier system including electron-electron interaction. We find that the in-plane magnetic field dependence of the Hall coefficient is suppressed by the weakening of screening and the electronelectron interaction. However, the in-plane magnetoresistance is mostly determined by the change of the screening of the system, and can therefore be strongly field dependent.

PACS Number : 72.25.Rb; 72.15.Gd; 73.40.Qv
\end{abstract}

The phenomena of apparent two dimensional (2D) metallic behavior and the associated 2D metal-insulator transition (MIT) continue to attract a great deal of attention $[1,2]$. The low temperature resistivity $\rho(T)$, in zero applied field, shows remarkably strong "metallic"like (i.e. $d \rho / d T>0$ for $n>n_{c}$ ) temperature dependence for $2 \mathrm{D}$ carrier densities above the so-called critical carrier density $\left(n_{c}\right)$ for the 2D MIT whereas, for $n<n_{c}$, the system exhibits insulating behavior $(d \rho / d T<0)$. The application of an in-plane magnetic field $B$ has interesting effects on the 2D metallic phase, i.e., at a fixed low $T$ the system develops a large positive magnetoresistance with $\rho(B)$ increasing very strongly (by as much as a factor of 4 ) with $B$ upto a maximum field $B_{s}$, and for $B>B_{s}, \rho(B)$ either saturates (or increases slowly with $B$ for $B>B_{s}$ ) showing a distinct kink at $B=B_{s}$ [3]. The observed temperature, density, and parallel magnetic dependence of the 2D "metallic" resistivity $[2,4]$ can be explained by the screening theory in which the strongly temperature dependent effective screened charged impurity disorder is the qualitative reason underlying the striking metallic behavior of dilute 2D carrier systems.

Remarkably, recent measurements of the 2D Hall resistance [5] in a parallel magnetic field have shown unexpected physical behavior which is in sharp contrast with the strong in-plane field dependence of the 2D magnetoresistivity. The measured Hall coefficient seems to contradict qualitatively the results based on the screening theory [6] even though the longitudinal magnetoresistance can be explained by the change of the screening as the spin-polarization of the system varies. The measured Hall coefficient is found not to vary with parallel magnetic field (or spin-polarization) for fields ranging from 0 to well above $B_{s}$, where $B_{s}$ is the complete spin-polarization field. However, the screening theory shows very strong magnetic field (spin-polarization) dependence. Since the screening theory, at least in its most elementary formulation [6], cannot explain this unexpected Hall coefficient data Vitkalov et al. [5] proclaim that the electron-electron inter-subband scattering in the spin-polarized system is the main reason for the experimental behavior. The qualitative disagreement between the experimental Hall data of ref. [5] and the screening theory [6] is therefore a problem in our fundamental understanding of $2 \mathrm{D}$ transport because the screening theory [7] can explain the temperature dependent Hall coefficient in p-GaAs [8] and Si-MOSFET [9].

Motivated by this puzzling experimental observation [5], we investigate in this paper, based on the screening model, the spin-polarization dependence of the weakfield Hall resistance and magnetoresistance. For complete comparison with the experimental Hall coefficient we include in our calculation the electron-electron scattering between two different spin subbands. In ref. [5] Vitkalov et al. compare the Hall coefficient data with the zero temperature results of screening theory [6] in the strong screening limits $q_{T F} / 2 k_{F} \gg 1$ (where $q_{T F}$ is the Thomas-Fermi screening wave vector and $k_{F}$ is the Fermi wave vector). Our calculation, which includes finite temperature and fully wave vector dependent screening, is in qualitative agreement with the experimental results [5] on the spin-polarization dependence of the weak field Hall coefficient although electron-electron scattering might play a role at finite temperature as we show. We therefore resolve the experimental problem posed in ref. [5].

To calculate the Hall coefficient and the magnetoresistance, we solve the coupled kinetic equations for two kinds of carriers. When the parallel magnetic field is applied to the system the electron densities $n_{ \pm}$for spin up/down are not equal with the total density $n=n_{+}+n_{-}$ fixed. The spin-polarized densities themselves are obtained from the relative shifts in the spin up and down bands introduced by the Zeeman splitting associated with the external applied field $B$. Since there are two groups of electrons (spin up and down) we need to consider inter-spin-band electron-electron scattering, which contributes to resistivity in addition to electron scattering by charged impurities and phonons. In the presence of an applied field, the carrier momentum will relax to 


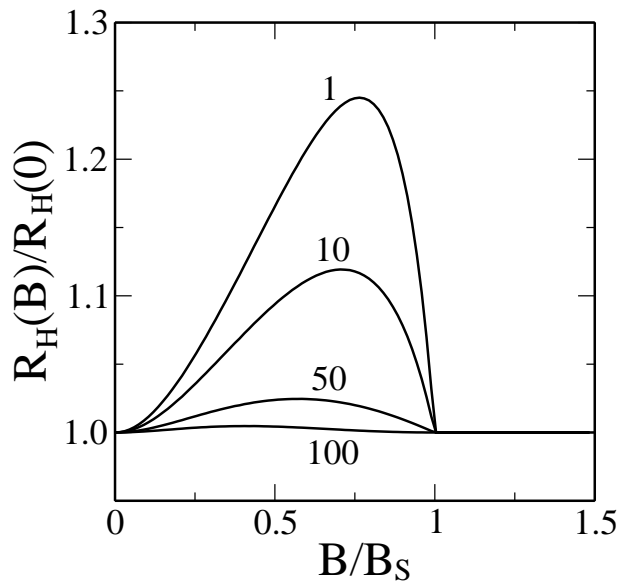

FIG. 1. Normalized Hall coefficient $R_{H}(B) / R_{H}(0)$ as a function of in plane magnetic field (spin polarization of the system) for different densities, $n=1,10,50,100 \times 10^{10} \mathrm{~cm}^{-2}$ and at $T=0 K$. At $B=B_{s}$ the 2 D system is completely spin-polarized.

equilibrium by electron-electron, electron-impurity, and electron-phonon scattering (which is neglected in this calculation because at low temperatures of interest to us phonon scattering is unimportant). The electronelectron relaxation rate $1 / \tau_{e e}$ will only affect the relative momentum (i.e. the electron-electron scattering for the same spin is neglected in this calculation). The electronelectron scattering relaxes the relative velocity or relative momentum between two different populations to zero. In the steady state, the kinetic equations of motion in the presence of an electric field $\mathbf{E}$ and magnetic field $\mathbf{B}$ for spin up/down electrons, taking into account the collisions with spin down/up electrons, have the form $(c=\hbar=1)$ [11]

$$
\begin{aligned}
& m_{1} \frac{\mathbf{v}_{1}}{\tau_{1}}+M \frac{n_{2}}{n} \frac{\mathbf{v}_{1}-\mathbf{v}_{\mathbf{2}}}{\tau_{e e}}=e \mathbf{E}+e\left(\mathbf{v}_{1} \times \mathbf{B}\right), \\
& m_{2} \frac{\mathbf{v}_{2}}{\tau_{2}}+M \frac{n_{1}}{n} \frac{\mathbf{v}_{2}-\mathbf{v}_{\mathbf{1}}}{\tau_{e e}}=e \mathbf{E}+e\left(\mathbf{v}_{2} \times \mathbf{B}\right),
\end{aligned}
$$

where $m_{i}$ is the effective mass $(i=1,2$ denotes up/down spin subbands) for each group, $M=n m_{1} m_{2} /\left(m_{1} n_{1}+\right.$ $m_{2} n_{2}$ ), and $\tau_{i}$ is the (energy and temperature dependent) 2D carrier transport scattering time (the so-called momentum relaxation time) determined by the screened charged impurity scattering [10] and $\tau_{e e}$ is the electronelectron relaxation time for the relative momentum of the spin polarized system.

By solving the system of equation for $\mathbf{v}_{i}$ and substituting these velocities into expression for the current density $\mathbf{j}=n_{+} e \mathbf{v}_{+}+n_{2} e \mathbf{v}_{-}$, we find the resistivities $\rho_{x x}$ and $\rho_{x y}$,

$$
\begin{gathered}
\rho_{x x}=\frac{1}{n e} \frac{\langle\tilde{\mu}\rangle\left[1+\frac{n_{1} \mu_{2}+n_{2} \mu_{1}}{n \mu_{e e}}\right]+\frac{\mu_{1} \mu_{2}\left(n_{1} \mu_{2}+n_{2} \mu_{1}\right)}{n} B_{z}^{2}}{\left[\langle\mu\rangle+\mu_{1} \mu_{2} / \mu_{e e}\right]^{2}+\left(\mu_{1} \mu_{2} B_{z}\right)^{2}}, \\
\rho_{x y}=\frac{B_{z}}{n e}\left\langle r_{H}\right\rangle,
\end{gathered}
$$

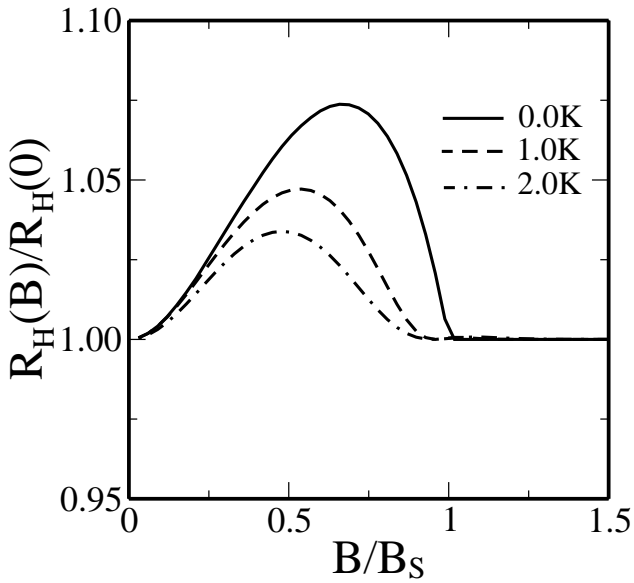

FIG. 2. Calculated Hall coefficient $R_{H}(B) / R_{H}(0)$ as a function of in plane magnetic field (spin polarization of the system) for different temperatures, $T=0,1,2 \mathrm{~K}$, and $n=20 \times 10^{10} \mathrm{~cm}^{-2}$.

where $\langle\tilde{\mu}\rangle=\langle\mu\rangle+\mu_{1} \mu_{2} / \mu_{e e}$ and $B_{z}$ is the applied magnetic field normal to the $2 \mathrm{D}$ layer and $r_{H}$, the so-called Hall ratio, is given by

$$
\left\langle r_{H}\right\rangle=1+\frac{\left\langle\mu^{2}\right\rangle-\langle\mu\rangle^{2}}{\left[\langle\mu\rangle+\mu_{1} \mu_{2} / \mu_{e e}\right]^{2}+\left(\mu_{1} \mu_{2} B_{z}\right)^{2}} .
$$

Here $\mu_{i}=e \tau_{i} / m_{i}$ and $\mu_{e e}=e \tau_{e e} / M$ and the average mobility is defined by $\langle\mu\rangle=\left(n_{1} \mu_{1}+n_{2} \mu_{2}\right) / n$ and $\left\langle\mu^{2}\right\rangle=\left(n_{1} \mu_{1}^{2}+n_{2} \mu_{2}^{2}\right) / n$. When inter-subband electronelectron scattering is weaker than the impurity transport scattering times $\left(\tau_{e e} \gg \tau_{i}\right)$ we have $\rho_{x x}=1 / n e\langle\mu\rangle$ and $\left\langle r_{H}\right\rangle=\left\langle\mu^{2}\right\rangle /\langle\mu\rangle^{2}$. In the other limit, $\tau_{e e} \ll \tau_{i}$, we have $\rho_{x x}=\left(n_{1} / n \mu_{1}+n_{2} / n \mu_{2}\right) / n e$ and $\left\langle r_{H}\right\rangle \rightarrow 1$. These equations immediately imply that the Hall coefficient will have very weak temperature dependence (i.e. $r_{H} \sim 1$ ) when the inter-spin subband electron-electron scattering dominates over the impurity scattering.

In Figs. 1 and 2 we show our calculated Hall coefficient without inter-subband electron-electron scattering. In this case the total conductivity is the sum of the conductivities of each group, $\sigma=\sigma_{1}+\sigma_{2}$, and the Hall coefficient is given by $\left\langle r_{H}\right\rangle=\left\langle\mu^{2}\right\rangle /\langle\mu\rangle^{2}$. Throughout this paper we use the parameters corresponding to SiMOSFET electron systems following ref. [5]. In Fig. 1 we show our calculated Hall coefficient, $R_{H}(B) / R_{H}(0)$, $\left(R_{H}=\left\langle r_{H}\right\rangle / n e\right)$ for several carrier densities, $n=1,10$, $50,100 \times 10^{10} \mathrm{~cm}^{-2}$ (which correspond to the screening strength $q_{T F} / 2 k_{F}=35,11,5,3.5$ respectively) as a function of in-plane magnetic fields at $T=0$. As inplane magnetic field increases the spins are polarized, and at $B=B_{s}$ the system is completely spin-polarized. In the low density limit (strong screening, $q_{T F} / 2 k_{F} \gg 1$ ) the normalized Hall coefficient is strongly dependent on the polarization of the system. But for high densities (weak screening) the coefficient is almost independent of the spin polarization. Thus the polarization dependent 


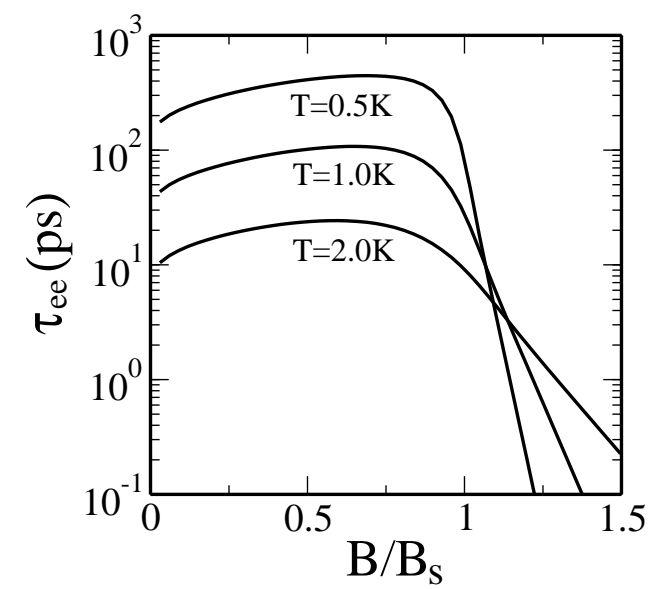

FIG. 3. Calculated inter spin-subband electron-electron scattering times for different temperatures $T=0.5,1.0,2.0 \mathrm{~K}$ as a function of the parallel magnetic field. We use the $2 \mathrm{D}$ density $n=20 \times 10^{10} \mathrm{~cm}^{-2}$.

Hall coefficient is suppressed as screening effects decrease. We find that the normalized Hall coefficient for a density $n=25 \times 10^{10} \mathrm{~cm}^{-2}$ (corresponding to ref. [5]) increases only about $6 \%$ at most as the system gets fully spinpolarized by the applied field.

Since the screening function is suppressed by thermal effects we expect the Hall coefficient to be suppressed at finite temperatures. In Fig. 2 we show our calculated Hall coefficient, $R_{H}(B)$, for several temperatures, $T=0$, $1,2 \mathrm{~K}$ and a fixed density $n=20 \times 10^{10} \mathrm{~cm}^{-2}$. As the temperature increases the normalized Hall coefficient shows suppressed field dependence mostly due to the weakening of the screening. Comparison between our results and the experimental results [5] shows good qualitative agreement. Thus, the observed field independence of the Hall coefficient can be qualitatively explained by the screening theory. However, in ref. [5] Vikalov et al. conclude, by comparing their measured Hall coefficient (which does not vary with parallel magnetic field) with the theoretical expectations based on the screening theory [6] calculated in the strong screening limit $\left(q_{T F} / k_{F} \gg 1\right)$ and at zero temperature, that the screening theory disagrees qualitatively with the experimental Hall effect results, and therefore the strong electron-electron scattering is a possible explanation for their data. In order to investigate the effects of electron-electron scattering on the Hall coefficient we consider fully Eq. (4) which includes electron-electron scattering as well as the screened charged impurities. We explicitly calculate the electron-electron scattering time $\tau_{e e}$ defined as the relaxation time of the relative momentum between spin up and down carriers [12]. For an unequal spin population we have $\tau_{e e}$

$$
\frac{1}{\tau_{e e}}=\frac{8\left(k_{B} T\right)^{2}}{3(\pi)^{2}} \frac{n m^{3}}{n_{1} n_{2}} p \int_{0}^{\pi} d \theta \sin \theta\left|\frac{2 \pi e^{2}}{q \varepsilon(q)}\right|^{2}[f(p)]^{2}
$$

where $f(p)=\left(1+p^{2}+2 p \cos \theta\right)^{1 / 2}$ with $p=\left(n_{1} / n_{2}\right)^{1 / 2}$,

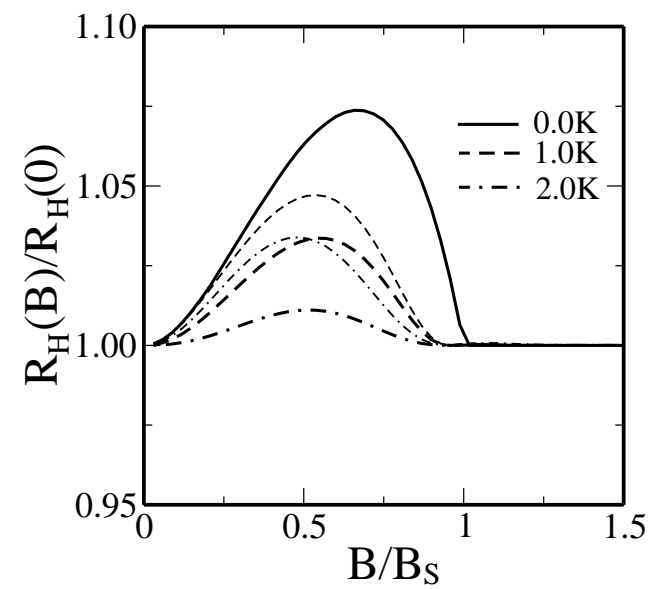

FIG. 4. Calculated Hall coefficient $R_{H}(B) / R_{H}(0)$ as a function of in plane magnetic field (spin polarization of the system) for different temperatures, $T=0,1,2 \mathrm{~K}$, and $n=20 \times 10^{10} \mathrm{~cm}^{-2}$. Thick (thin) lines indicate the results with (without) electron-electron scattering.

$q=4 \pi \sqrt{n_{1} n_{2}} \sin (\theta) / f(p)$, and $\varepsilon(q)$ is the total dielectric function of the system. Thus we have that $\tau_{e e}^{-1} \propto T^{2}$. In the very low temperature limit we expect the contribution of the electron-electron scattering to the Hall coefficient to be negligible because of this $T^{2}$ dependence of $\tau_{e e}^{-1}$. At low temperature, therefore $\left(T \ll T_{F}\right)$ our results (Figs. 1 and 2) neglecting $\tau_{e e}$ effects apply.

In Fig. 3 we show our calculated inter spin-subband electron-electron scattering times, $\tau_{e e}$, as a function of the parallel field (or spin-polarization). Our results show that $\tau_{e e}$ depends strongly on the spin-polarization and temperature. (Note in ref. [5] a constant parameter $\tau_{e e}$ is used to fit the Hall coefficient data, which is incorrect.) In general, we find the elastic scattering time due to ionized impurities at the interface to be $\tau_{i} \approx 7 \mathrm{ps}$ with an impurity density $n_{i}=3 \times 10^{10} \mathrm{~cm}^{-2}$, which corresponds to the experimental Si-MOSFET sample of the mobility $\mu \approx 2 \times 10^{4} \mathrm{~V} / \mathrm{cm}^{2} \mathrm{~s}$. Thus the calculated $\tau_{e e}$ is much larger than the elastic scattering time $\tau_{i}$ in the low temperature limit where the $2 \mathrm{D}$ experiments are typically carried out.

With the results in Fig. 3 we calculate the Hall coefficient of the spin-polarized system including electronelectron scattering. Fig. 4 shows the calculated Hall coefficient $R_{H}(B) / R_{H}(0)$ as a function of in plane magnetic field for different temperatures, $T=0,1,2 \mathrm{~K}$, and $n=20 \times 10^{10} \mathrm{~cm}^{-2}$. Our calculation shows that the electron-electron scattering leads to the further suppression in the field dependence of the Hall coefficient, leading to even better agreement between experiment [5] and our theory.

Fig. 5 shows the calculated magnetoresistance as a function of in-plane magnetic field for different temperatures and a carrier density $n=2 \times 10^{11} \mathrm{~cm}^{-2}$. This strong positive magnetoresistance can be explained by the sys- 


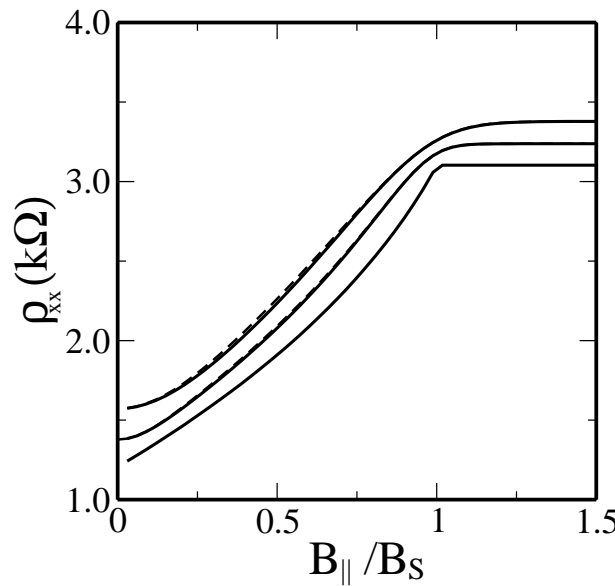

FIG. 5. Calculated magnetoresistance as a function of in-plane magnetic field for different temperatures, $\mathrm{T}=0,1,2 \mathrm{~K}$ (from bottom to top). The results are shown for the carrier density $n=2 \times 10^{11} \mathrm{~cm}^{-2}$. The solid (dashed) lines indicate calculated results without (with) electron-electron inter-spin subband scattering.

tematic suppression of screening as the spin-polarization of the system changes $[4,6]$. The saturation of the magnetoresistance above $B_{s}$ is attributed to the complete spinpolarization of the system. Considering electron-electron scattering in the calculation produces very small quantitative modification in the magnetoresistance even though the electron-electron scattering times are comparable to the elastic impurity scattering times (at $\mathrm{T}=2 \mathrm{~K}$ ). In Fig. 5 the solid (dashed) lines indicate calculated results without (with) electron-electron interaction. Even though the electron-electron scattering rate is stronger than the elastic scattering rate, the calculated magnetoresistance shows the same behavior. Thus, the experimentally measured strong positive magnetoresistance with increasing in-plane magnetic field is induced mostly by the change of the screening properties, not by the inter-spin subband electron-electron scattering.

In Fig. 6 we show that calculated longitudinal magnetoresistance $\rho_{x x}$ as a function of perpendicular magnetic field $\left(B_{z}\right)$ for different temperatures, $\mathrm{T}=0,1,2 \mathrm{~K}$. The inplane parallel magnetic field solely gives rise to the spin polarization of the system. The results are shown for a carrier density $n=2 \times 10^{11} \mathrm{~cm}^{-2}$ and a fixed in-plane magnetic field $B_{\|}=0.5 B_{s}$. For an unpolarized system (i.e., a single subband with an isotropic scattering rate) classical transport theory predicts no magnetoresistance (horizontal solid line in Fig. 6) since the Hall field exactly compensates the Lorentz force and the carriers drift in the direction of the applied field. In fact, single subband systems typically exhibit a negative magnetoresistance due to quantum corrections arising from weak localization or electron-electron interactions [13]. For a system with two different Fermi wave vectors (two occupied subbands) the classical transport theory predicts a positive magnetoresistance varying quadratically with magnetic

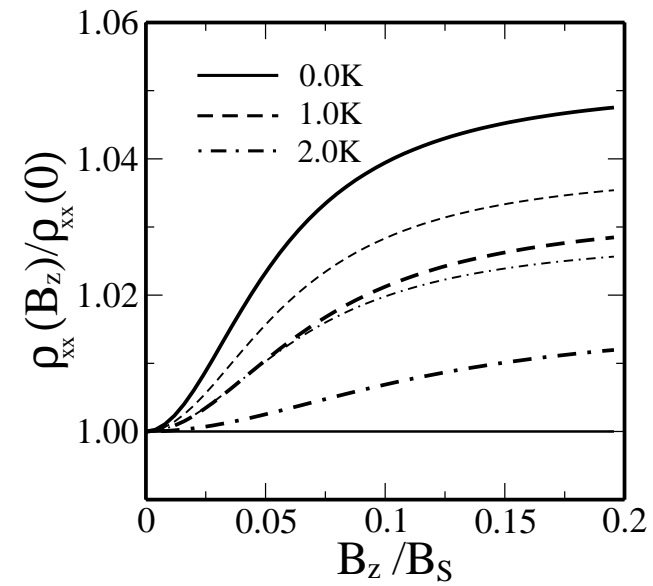

FIG. 6. Calculated magnetoresistance as a function of perpendicular magnetic field $\left(B_{z}\right)$ to the $2 \mathrm{D}$ plane for different temperatures, $\mathrm{T}=0,1,2 \mathrm{~K}$ (from bottom). The results are shown for the carrier density $n=2 \times 10^{11} \mathrm{~cm}^{-2}$ and a fixed in-plane magnetic field $B_{\|}=0.5 B_{s}$. The thick (dashed) lines represent results with (without) electron-electron interaction.

field $B_{z}$ at low fields and saturating at higher fields. In Fig. 6 we have the positive magnetoresistance as the magnetic field increases for a spin-polarized system. The positive magnetoresistance is again reduced by thermal effects (weakening of screening), and also by the electronelectron scattering (assuming that there are no quantum corrections).

In conclusion, we calculate the weak-field Hall coefficient and the magnetoresistance of a spin polarized system based on the screening theory. We find that the spinpolarization dependence of the Hall coefficient is strongly suppressed by the weakening of screening. The electronelectron inter-spin subband scattering gives rise to an additional suppression of the Hall coefficient, but in the low temperature experimental regime the inter-spin subband electron-electron scattering is not quantitatively important. Our theory provides a very good explanation for recent experiments [5] on the magnetic field dependence of the 2D Hall coefficient.

This work is supported by US-ONR, NSF, and LPS.

[1] E. Abrahams, S. V. Kravchenko, and M. P. Sarachik, Rev. Mod. Phys. 73, 251 (2001); S. V. Kravchenko and M. P. Sarachik, Rep. Prog. Phys. 67, 1 (2004)

[2] S. Das Sarma and E. H. Hwang, Solid State Comm. 135, 579 (2005).

[3] T. Okamoto, K. Hosoya, S. Kawaji, and A. Yagi, Phys. Rev. Lett. 82, 3875 (1999).

[4] S. Das Sarma and E. H. Hwang, Phys. Rev. B 72, 205303 (2005).

[5] S. A. Vitkalov et al., Phys. Rev. B 63, 193304 (2001); S. 
A. Vitkalov, Phys. Rev. B 64, 195336 (2001).

[6] V. T. Dolgopolov and A. Gold, JETP Lett. 71, 27 (2000).

[7] S. Das Sarma and E. H. Hwang, Phys. Rev. Lett. 95, 016401 (2005).

[8] X. P. A. Gao et al., Phys. Rev. Lett. 93, 256402 (2004).

[9] A. Yu. Kuntsevich et al., Pis'ma Zh. Eksp. Teor. Fiz. 81, 502 (2005); cond-mat/0504475 (2005).

[10] S. Das Sarma and E. H. Hwang, Phys. Rev. Lett. 83, 164 (1999); Phys. Rev. B 69, 195305 (2004).

[11] C. A. Kukkonen and P. F. Maldague, Phys. Rev. B 19, 2394 (1979).

[12] J. Appel and A. W. Overhauser, Phys. Rev. B 18, 758 (1978).

[13] P. A. Lee and T. V. Ramakrishnan, Rev. Mod. Phys. 57, 287 (1985); D. Belitz and T. R. Kirkpatrick, Rev. Mod. Phys. 66, 261-380 (1994). 